\title{
Lessons Learned from Co-Design and Co-Production in a Portable Health Clinic Research Project in Jaipur District, India (2016-2018)
}

\author{
Fumihiko Yokota ${ }^{1, *}$, Manish Biyani ${ }^{2}{ }^{3}$, Rafiqul Islam ${ }^{3}$, Ashir Ahmed ${ }^{4}$, Mariko Nishikitani ${ }^{1}$, \\ Kimiyo Kikuchi ${ }^{1}$, Yasunobu Nohara ${ }^{3} \mathbb{D}$ and Naoki Nakashima ${ }^{3}$ \\ 1 Institute of Decision Science for Sustainable Society, Kyushu University, Fukuoka 8190395, Japan; \\ makorin@med.kyushu-u.ac.jp (M.N.); kikuchi.kimiyo.715@m.kyushu-u.ac.jp (K.K.) \\ 2 Department of Nursing, Biyani Group of Colleges, Jaipur, Rajasthan 302039, India; \\ drmanishbiyani@gmail.com \\ 3 Medical Information Center, Kyushu University Hospital, Fukuoka 8128582, Japan; \\ rimaruf@med.kyushu-u.ac.jp (R.I.); y-nohara@info.med.kyushu-u.ac.jp (Y.N.); \\ nnaoki@med.kyushu-u.ac.jp (N.N.) \\ 4 Graduate School of Information Science and Electrical Engineering, Kyushu University, \\ Fukuoka 8190395, Japan; ashir@ait.kyushu-u.ac.jp \\ * Correspondence: yokota.fumihiko.785@m.kyushu-u.ac.jp; Tel.: +81-92-802-6052
}

Received: 6 September 2018; Accepted: 2 November 2018; Published: 12 November 2018

\begin{abstract}
Co-design and co-production with non-academic stakeholders has been recognized as a key approach in transdisciplinary sustainability research. The majority of transdisciplinary studies have been conducted in Europe and North America, with a marked lack of such research in the Asian context-particularly with regard to healthcare. Utilizing a case study involving mobile health check-ups performed using a portable health clinic system in Jaipur, India, from March 2016 to March 2018, this study identifies key factors in co-design and co-production that should be considered to ensure the project's sustainability. Thoroughly reviewing all of the documents and materials related to the case study's co-design and co-production, this study identifies the following key factors: (1) mutual stakeholder agreement on a long-term research plan, protocol, and budget; (2) harmonizing research objectives, frames, and the scale of stakeholder expectations; (3) stakeholders' commitment and a sense of ownership derived from their needs and priorities; (4) stakeholder trust; (5) effective coordinators; (6) personality type and characteristics of stakeholder leaders; (7) capacity building and the empowerment of local research staff and participants; and (8) continuous efforts to involve stakeholders throughout the co-design and co-production processes. Facilitating effective co-design and co-production, these factors will help ensure the future sustainability of projects.
\end{abstract}

Keywords: co-design and co-production; community-based; healthcare services; transdisciplinary research; India

\section{Introduction}

Involving both academic and non-academic stakeholders-such as governments, industries, and community members-co-design and co-production has been recognized as a key approach in transdisciplinary research [1-4]. This approach has become increasingly popular because it helps achieve sustainable social impact $[2,3,5,6]$. Moreover, this approach enhances the research process within the academic sphere of the social and natural sciences, as well as in terms of research practice and policy interrelationships [5-7]. Indeed, this approach is well-suited to transdisciplinary research, which favors enhanced knowledge, collaboration, and communication for sustainable 
research [1]. As such, adopted approaches tend to involve a mutual learning process and joint partnerships between interdisciplinary scholars and non-academic stakeholders from the fields of science, industry, politics, technology, and civil society [1,8,9]. Exemplifying the practical utility of such an approach, an international research program called "Future Earth" was launched at the United Nations (UN) Conference on Sustainable Development in June 2012. Incorporating the natural and social sciences to solve global environmental issues [10], Future Earth has "pioneered approaches to the co-design and co-production of solutions-oriented, transdisciplinary research for global sustainable development" [11].

However, transdisciplinary research remains hampered in a number of ways. First, the most recent reviews of transdisciplinary case studies have concluded that the methods and concepts of co-design and co-production processes lack clarity $[1,2,4,12]$. Second, the majority of extant transdisciplinary research only describes the early stages of co-design, rather than its actual implementation and application [2,7]. Indeed, which studies include key components of early co-design—such as the "framing of problems" [4,12,13], "social capital and partnerships with mutual trust" [14-16], "scaling" [17], "accountability" [9,18], "ownership" [18], as well as "priorities and needs" [1,9]—few have used case studies to discuss both co-design and co-production in a comprehensive, bottom-up manner [4]. Third, in comparison to Europe and North America, markedly few transdisciplinary case studies have been conducted in low-middle income countries in Asia-particularly in terms of health issues [2]. As such, key factors that positively influence the co-design and co-production of health-related transdisciplinary research in Asia remain vague and largely unknown.

Addressing these gaps, this paper uses a case study to discuss co-production and co-design in an Asian, health-related context. As part of the collaborative Future Earth project, a community-based, mobile health check-up research project called the Portable Health Clinic (PHC) was introduced to the Jaipur District, India, in March 2016. Several institutions were involved in this initiative, namely: Kyushu University (KU), the Biyani Group of Colleges (BGC), and Grameen Communications (GC). This paper discusses the lessons learned from this ongoing transdisciplinary initiative between March 2016 and March 2018. Based on the lessons learned, the study identifies key factors that facilitate the effective co-design and co-production of a sustainable PHC health check-up system. In addition, this paper aims to develop a methodological and conceptual framework of health-focused, transdisciplinary research in the Asian context, as well as a new model for collaboration.

\section{Operational Definitions and Methodology}

\subsection{Operational Definitions of Transdisciplinary Research, Co-Design, and Co-Production}

Given the broad and complex nature of real-world problems, transdisciplinary research has accumulated a variety of terms or names, including "interdisciplinary participatory research", "participatory action research", "multi-disciplinary participatory research", and "community-based participatory research (CBPR)" $[1-3,5,18]$. Based on commonalities between extant studies in this field $[1,3,5,7,8,18-20]$, this paper defines transdisciplinary research as "real-world, solutions-oriented research for a sustainable society, involving both academic and non-academic stakeholders such as governments, industries, and civil society organizations." Co-design is "a process of joint development and mutual learning with all stakeholders regarding the research plan and protocols including research objectives, design, and methods" [5,12]. Co-production is "a process of generating collaborative knowledge including implementation, analysis, capacity-building, and empowerment activities, engaging with both academic and non-academic stakeholders" [3,9].

\subsection{Data sources, Collection Procedures, and Analysis}

The data sources for this study comprised research reports, activity logbooks, meeting minutes, as well as the research plan and protocol (including a research timeline, activity schedules, and budgets). These documents were developed, reviewed, and revised multiple times with stakeholders during 
the co-design and co-production stage. During these processes, a number of meetings, consultations, and discussions were held with each stakeholder. In addition, two training sessions, six workshops, and six seminars were conducted with stakeholders. The content of these collaborative activities was documented in research reports, activity logbooks, and meeting minutes. These documents were coded and classified under several categories based on the lessons learned from co-design and co-production.

\section{The PHC Research Project in Jaipur: Four Phases, March 2016-March 2018}

Implemented in Bangladesh in 2010, the Portable Heath Clinic (PHC) - a remote healthcare system - was developed under the 2007 joint research agreement between GC and KU. A non-profit information technology (IT) company, GC is part of the Grameen Family of Organizations established by Dr. Muhammad Yunus, the Nobel Peace prize laureate and founder of Grameen Bank. Located in Bangladesh, GC provides software products, internet, hardware, networking services, and IT education [21]. An e-health service system, the PHC comprises a briefcase containing a set of medical sensor devices, allowing for mobile health check-ups and Skype telemedicine services in remote rural areas [22-26] (Figure 1). After KU Hospital joined the PHC project in 2012, the PHC focused on the prevention and management of non-communicable diseases (NCDs) such as diabetes, hypertension, and obesity. As of January 2018, more than 41,000 people at 32 locations in Bangladesh have used PHC services [25].

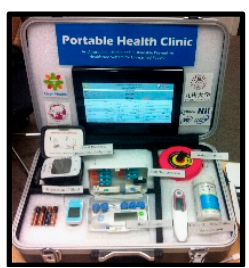

(a)

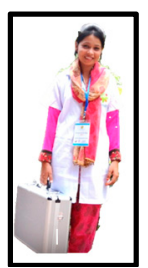

(b)

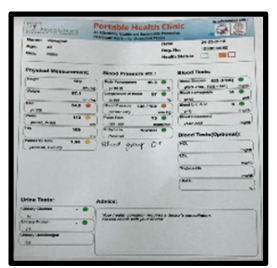

(c)

Figure 1. (a) Portable health clinic box; (b) healthcare entrepreneur; and (c) results of health check-ups.

\subsection{Phase 0: Co-Design and Co-Production Processes among KU, GC, and the BGC}

Part of the Future Earth project, the PHC project was initiated in India in March 2016 as a community-based health study co-operatively run by KU, GC, and the BGC (Figure 2). In addition to funding from KU's Institute of Decision Science for a Sustainable Society, KU provided technical support for the research design, methodology, and data analysis-which was conducted from interdisciplinary perspectives such as clinical medicine, public health, nursing, the social sciences, as well as information and communications technology (ICT). GC trained healthcare workers to use the PHC system software and services. The BGC served as a community coordinator linking KU and GC with local stakeholders in India, which providing human and facility resources for field research. The BGC is a non-profit educational organization that is committed to improving girls' education and empowerment in the Indian state of Rajasthan. Founded in 1997, the BGC has two campuses with more than 4000 students majoring in science, management, engineering, technology, and nursing. The BGC's initial collaboration began in 2012, when a BGC research and development director expressed strong interest in the PHC. 


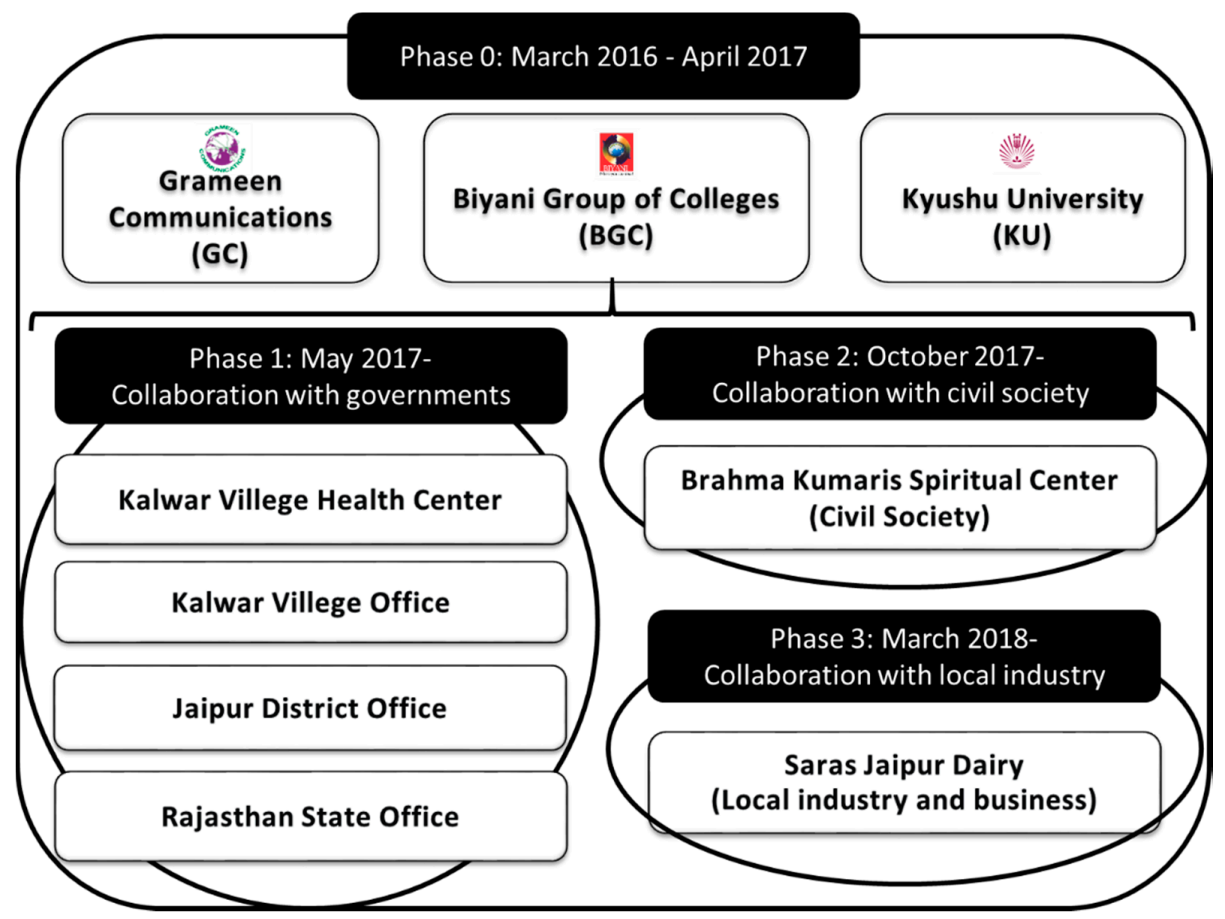

Figure 2. Stakeholders for the Portable Health Clinic (PHC) research project in Jaipur across the three phases (March 2016-March 2018).

During Phase 0, the PHC aimed to provide mobile health check-ups as a small-scale pilot project in order to understand the current health conditions among targeted rural communities in Jaipur District. At this stage, the project was designed to reduce the prevalence and incidence of NCDs such as diabetes, hypertension, and obesity among rural communities through sustainable co-operation with local government agencies and communities. In the longer term, the project sought to improve the health status of rural groups by establishing a sustainable healthcare business model in Jaipur District. These objectives and vision were shared and agreed upon by KU, GC, and the BGC to achieve some of the UN's Sustainable Development Goals (SDGs), including "good health and well-being" (SDG3) and "partnerships for the goals" (SDG17). However, a detailed long-term research plan, protocol, and budget were not developed at this early phase due to the uncertainty regarding funding and the absence of an official collaboration agreement between KU and the BGC.

In early February 2016, several Skype meetings and email exchanges were conducted between $\mathrm{KU}$, the BGC, and GC to create a research activity plan and organize a joint PHC staff training initiative in Jaipur. Consequently, a five-day training session was conducted in early March 2017 at the BGC's Jaipur campus. Three PHC trainers from GC, three staff members from KU, and more than 20 nursing students and instructors from the BGC participated in the training initiative. Thereafter, between March 2016 and March 2017, PHC mobile health check-up services were implemented on an irregular basis at BGC's two campuses. These services were free of charge. A total of 437 people (local residents, BGC staff, and students older than 18) voluntarily participated in the PHC project. While the services were predominantly arranged, advertised, and conducted by local trained BGC staff, KU and GU staff occasionally visited the campus to monitor the implementation process.

Basic participant demographic characteristics (such as age, sex, and education level) were collected using a standardized registration sheet. The following anthropometric and clinical data were measured or tested during an individual's check-up: (1) height; (2) weight; (3) hip circumference; (4) waist circumference; (5) body temperature; (6) systolic blood pressure; (7) diastolic blood pressure; (8) blood glucose; (9) blood hemoglobin; (10) urinary glucose; (11) urinary protein; (12) blood cholesterol; (13) blood uric acid; (14) pulse rate; and (15) blood type. Based on the results of each test, the participant's health status was categorized into one of four color-coded risk levels: (1) green (healthy); (2) yellow 
(caution); (3) orange (affected); and (4) red (emergent). These data were analyzed by the BGC coordinator and KU research staff and presented at the annual BGC conference in Jaipur in October 2017, as well as at the Future Earth conference in Fukuoka, Japan, in December 2017. In summation: during Phase 0, KU, GC, and the BGC conducted the pilot studies, developed the research plan, carried out training, implemented the research, analyzed the data, and determined the results from a feedback workshop (Figure 3).
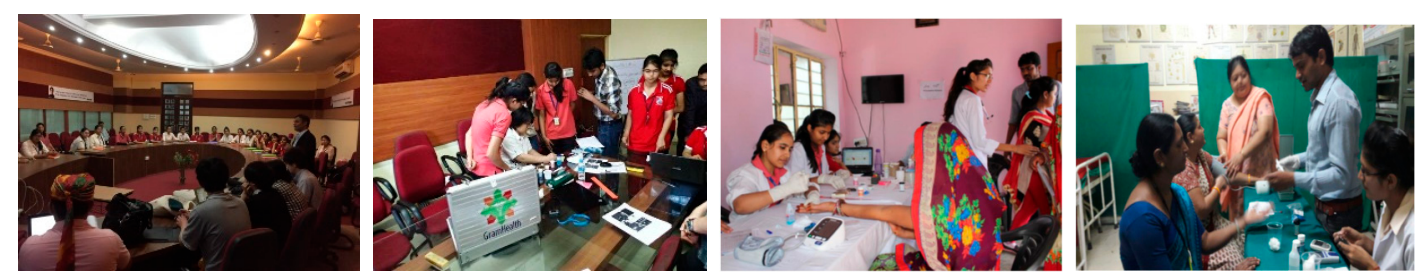

Figure 3. Initial meeting, staff training, and pilot implementation jointly conducted by Kyushu University (KU), Grameen Communication (GC), and the Biyani Group of Colleges (BGC) during Phase 0 (March 2016-April 2017).

\subsection{Phase 1: Co-Design Process with Local Government Agencies}

During Phase 0-that is, the pilot phase-KU and the BGC realized that permission and approval from local government agencies would be necessary to officially implement the PHC project in Rajasthan State (Figure 4). Consequently, the KU and BGC research teams visited the following agencies in May 2017: the Government of Rajasthan's Department of Medical, Health, and Family Welfare; the Kalwar Village Office; and the Kalwar Health Center (Figure 2). This process was greatly facilitated by the BGC having pre-established relationships with the mayor of Kalwar Village and a director of Rajasthan's Department of Medical, Health, and Family Welfare. At their initial meeting with government stakeholders, the KU and BGC research teams explained the visions, objectives, and activities of the PHC project. Their priorities, needs, and roles were then discussed, resulting in six changes to the project. First, the government stakeholders requested that several health check-up components be added to the, namely maternal and child health $(\mathrm{MCH})$, as well as oral health. Similar to NCDs, these are significant health issues in both Rajasthan and greater India. Second, they requested that PHC services be provided to community residents free of charge for the first year in order to gain their trust and satisfaction. Third, the government stakeholders requested that the project be used to promote health awareness - especially disease prevention-since the majority of citizens do not seek healthcare services unless they have severe symptoms or pain. Fourth, the stakeholders requested the production of data-based evidence that PHC services are effective in changing people's health behaviors and preventing the spread of disease. A time period of two years to gather data was suggested. Fifth, they advanced the need to make the project replicable in other villages and districts in Rajasthan, thus ensuring that the project can be scaled up. Finally, the government stakeholders requested that the project be aligned with India's national health policy framework and mission.

Under these conditions, the government stakeholders authorized the implementation of the PHC project in Kalwar Village. Following this initial meeting and consultation, the BGC and KU research teams had additional face-to-face meetings with the village's mayor and a medical doctor at Kalwar Heath Center to re-design the PHC research plan and protocol (Figure 2). Phase 1 also included an informal brainstorming dialogue with local Ayurvedic doctors-that is, practitioners of Ayurveda, a traditional Hindu system of medicine-and World Health Organization (WHO) officers to improve our research plan. Therefore, Phase 1 constituted the expansion of collaboration with both government and non-governmental stakeholders (Figure 5). While the project's research objectives, plan, and protocol were revised to reflect the requests of the government stakeholders, their first request proved problematic due to limited financial and human resources during Phase 1 . As such, the project did not add $\mathrm{MCH}$ and oral health check-ups to the PHC services at this stage. 


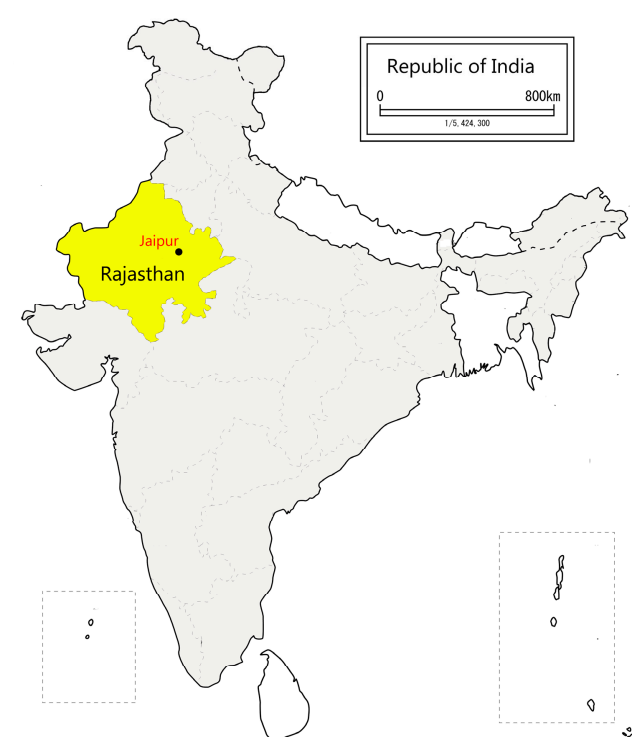

Figure 4. Location of Jaipur and Rajasthan.

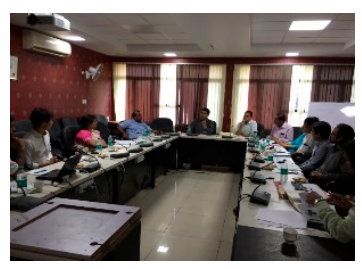

(a)

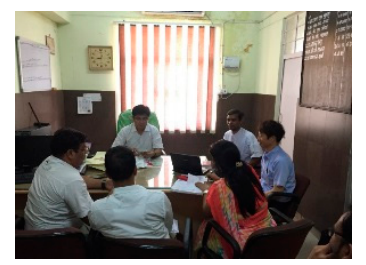

(b)

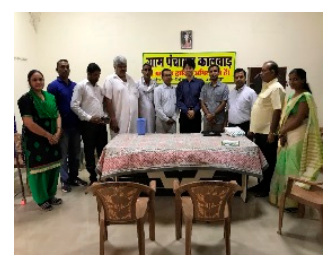

(c)

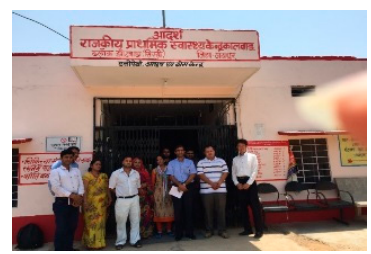

(d)

Figure 5. Courtesy visits (including meetings and discussions) to different levels of government during Phase 1 (May 2017-present). (a) The State of Rajasthan; (b) Jaipur District; (c) Kalwar Village Government Office; and (d) Kalwar Health Center.

\subsection{Phase 2: Co-Design and Co-Production with a Civil Society Organization}

Phase 2 began in October 2017, when KU and the BGC signed an official academic collaborative agreement. Based on the revised research plan and protocol (Phase 1), we decided to investigate the health needs, priorities, and longitudinal effects of the PHC services among different population cohorts in Jaipur District. To achieve these goals, it was necessary to provide check-ups to the same individuals over a period of time in order to monitor changes in their health status, behaviors, needs, and priorities. We recognized that it was easier to follow up with members of civil society organizations than with general community residents over the years. Consequently, the BGC first contacted Brahma Kumaris World Spiritual University (BKWSU) as a potential stakeholder able to provide a target population group (Figure 2).

Established in India during the 1930s, BKWSU is a spiritual civil society organization with more than 825,000 regular members in 100 countries [27]. In October 2017, the BGC and BKWSU signed a memorandum of understanding (MOU) in Jaipur. This process went smoothly because the BGC and BKWSU already had a good relationship as a result of previous collaboration in stress management activities, and both are committed to improving access to healthcare services in local communities. In November 2017, the BGC and BKWSU agreed to implement pilot PHC services at a BKWSU center located about 20 min away from the Jaipur city center. In addition, the Biyani Ethics Committee approved the PHC research project in Jaipur in December 2017.

In January 2018, the BGC, BKWSU, and KU held a joint three-day training session in Jaipur; the session was coordinated by the BGC. Following this session, a total of 91 participants voluntarily agreed to receive a PHC check-up and participated in a survey. A BGC research team-comprising one field research coordinator, five trained healthcare workers, five survey interviewers, two IT data 
managers, and three field supervisors-carried out PHC services for a period of two days at the BKWSU center. The team were joined by one field research supervisor and one data management specialist from KU. Three BKWSU staff assisted in selecting and organizing volunteers who were willing to participate in the health check-up and survey interview. The PHC research staff comprised students in BGC's nursing department, who were trained to provide PHC services in Hindi. Prior to implementing the PHC services, health awareness events were held at the BKWSU center to inform people about the availability of PHC services. The participants were regular BKWSU members older than 18 who were willing to participate. Project staff from BKWSU and the BGC scheduled their appointments. Throughout these co-design and co-production with BKWSU in Phase 2 (Figure 6), we identified their health needs for additional blood tests such as hemoglobin A1c (HbA1c), High Density Lipoprotein (HDL)/Low Density Lipoprotein (LDL) cholesterol, and triglycerides.
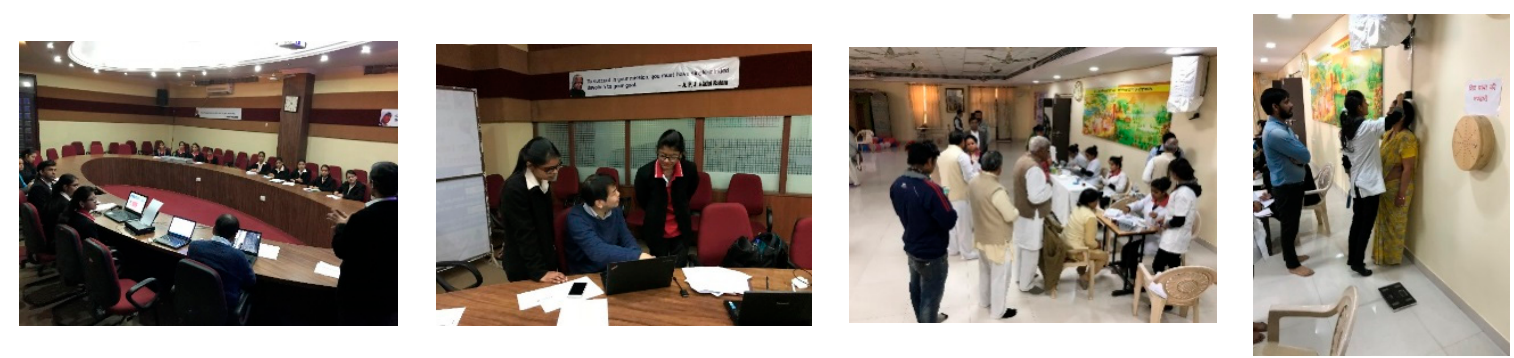

Figure 6. Seminars, workshop training, and pilot implementation jointly conducted by the KU, BGC, and Brahma Kumaris World Spiritual University (BKWSU) Center during Phase 2 (October 2017-present).

\subsection{Phase 3: Co-Design and Co-Production with a Local Industry}

Phase 3 started in January 2018. Sharing the same core research objective as Phase 2, Phase 3 sought to understand the health status, behaviors, and needs of local communities, as well as the effectiveness and acceptability of the PHC project in Jaipur District. However, unlike Phase 2, the target population in Phase 3 comprised industry employees rather than local residents or members of the civil society. Our aim was to identify the subpopulations that were most in need of services. Thus, Phase 3 enabled the comparison between the health needs, status, and behaviors of members of civil society organizations, industry employees, and general community members.

First, the BGC staff called local industries with whom they had a good relationship to schedule a meeting to explain our research. After several industries were contacted, one of the largest milk product enterprises in Rajasthan State, Saras Jaipur Dairy (SJD), expressed strong interest in providing health check-ups for their employees, and agreed to let us use their facility rooms in March 2018 (Figure 2). Located near the Jaipur city center, SJD is approximately a 40-minute drive from the BGC. SJD's expectation was that we would establish a sustainable healthcare service system for its employees.

In March 2018, the BGC and KU held a joint three-day training session at the BGC campus in Jaipur. At this time, new aspects of PHC services-namely, an eye exam, as well as blood tests for HbA1c, HDL/LDL cholesterol, and triglycerides-were added due to these items being in high demand during phases 1 and 2. These additional tests were made possible by the provision of additional financial support in Phase 3. For the eye check-up, we used a new portable eye camera recently introduced from Germany and produced by Bosch Eye Care (Robert Bosch Engineering and Business Solutions Pvt. Ltd., Bangalore, India) that could capture high-quality images of the eye fundus. This technology allows for easy screening to detect early signs of retinal abnormalities such as diabetic retinopathy, glaucoma, and age-related macular degeneration (AMD). For the hemoglobin test, we used a new device called the Astrim noninvasive blood vessel monitoring apparatus (Sysmex, Kobe, Japan).

After the training, a PHC camp was set up in the SJD facility space, and services were provided for a period of three days (Figure 7). The BGC research team was expanded to comprise one field research coordinator, three field supervisors, six trained healthcare workers, six survey interviewers, two IT data managers, two registration staff, four eye photo staff, and two medical doctors. Three staff 
from KU participated in both the training and field implementation to provide support and advice. A total of 205 people participated in both the check-up and survey interview. All of the participants were SJD employees from different sectors-including finance and accounting, factory production, marketing, engineering, security, cleaning, and transport-who were willing to participate. Following this three-day PHC camp at SJD, another round of PHC services was held on the BGC campus for one day. Approximately 87 BGC staff and faculty members participated.

As such, the process of PHC services provided at the SJD and BGC during Phase 3 was the same as that in Phase 2. However, Phase 3 investigated health needs from the perspective of service providers. The need for this qualitative investigation was based on key informant interviews with two local doctors, one pharmacist, and two nurses. The PHC project in Jaipur District remains ongoing, providing a basis for further sustainable transdisciplinary research.
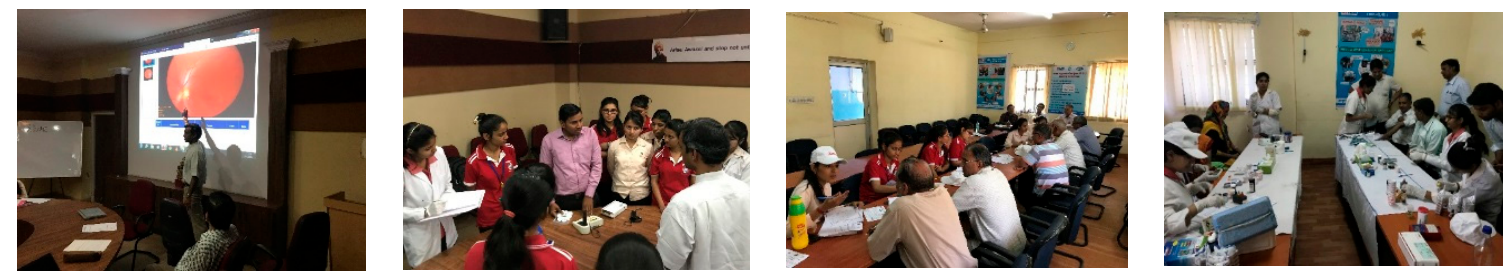

Figure 7. Seminars, training, and pilot implementation jointly conducted by the KU and BGC at the Saras Jaipur Dairy (SJD) and BGC sites during Phase 3 (March 2018-present).

\subsection{Summary of Co-Design and Co-Production in All Four Phases}

Six steps were followed in the four phases described in this section (Figure 8). These steps can be summarized as follows. Step 1: Jointly develop and revise a research plan and protocol that include research objectives, methods, a timeline of activities, and a budget. Step 2: Establish a collaborative agreement regarding the research plan and protocol with both academic and non-academic stakeholders through meetings, consultations, and workshops. Step 3: In collaboration with stakeholders, conduct local staff training based on the protocol in order to improve the quality of services and data. Step 4: Implement the pilot PHC project involving all of the stakeholders. Step 5: Analyze and disseminate the results obtained through feedback to all of the stakeholders by means of presentations, workshops, and conferences. Step 6: Revise the research plan and protocol based on feedback from stakeholders, including provision for local needs, as well as stakeholder priorities and requests.

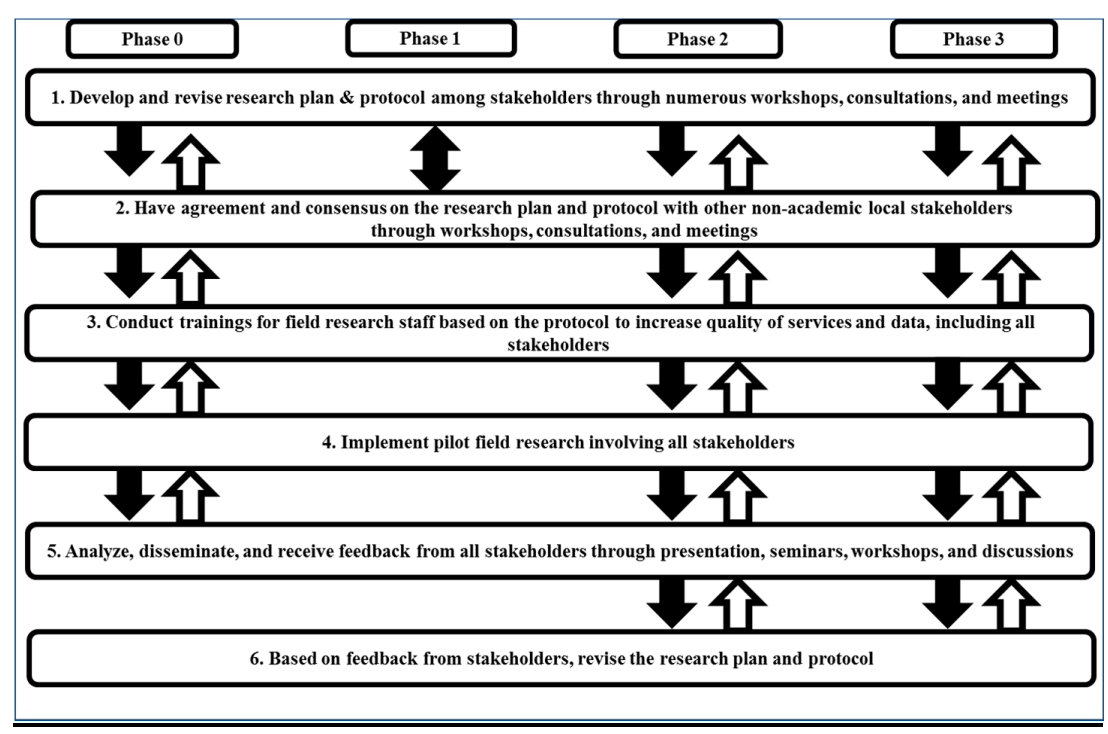

Figure 8. The co-design and co-production processes in the Portable Health Clinic (PHC) research project in India. 


\section{Lessons Learned and Key Factors for Effective Co-Design and Co-Production}

This section presents the lessons learned through the processes of co-design and co-production during the four phases discussed in the previous section. Based on these lessons learned, this section also presents the key factors that facilitated effective co-design and co-production (Figure 9). We identified eight key lessons and factors.

First, we learned that it is critical that stakeholders share, mutually agree on, and understand a long-term research plan, protocol, and budget (including a timeline, stakeholders' roles, and data ownership policy) to ensure effective co-design and co-production. We failed to develop a research plan and protocol in Phase 0 due to insufficient funds and manpower. Without a long-term plan with a secure budget, well-defined stakeholder roles, or a data ownership policy, we experienced difficulties insofar as stakeholders were unwilling to agree on collaborative implementation. These problems were resolved in Phase 2, when we jointly developed a research plan and protocol.

Second, we learned that stakeholder expectations need to correlate with research objectives, frames, and local or regional scales. Since this research was based on our proposal rather than stakeholder requests, we needed to engage with stakeholders to harmonize the research objectives, frames, and scales. In Phase 1, local government stakeholders expected that the PHC project would: (1) include $\mathrm{MCH}$ and oral health services; (2) gain the trust of local communities; (3) increase health awareness in local communities; (4) produce evidence that PHC services are effective; (5) be replicable in other villages or districts in Rajasthan for scale-up purposes; and (6) align with India's national health policy framework. At this stage, we were unable to integrate all of the stakeholder expectations into our research plan and protocol due to insufficient funding and a lack of human resources. Moreover, we did not have enough experience or evidence to prove that PHC services are an effective model that is able to gain a community's trust. Consequently, we needed to revise our research objectives to identify relevant or desired needs for check-ups, as well as investigate the effectiveness of PHC services on people's health and behavior over time among smaller-scale subpopulations in different parts of Jaipur.

Third, we learned that co-design and co-production are much more effective and smoothly implemented when local stakeholders are highly committed to the research and recognize their stake in the results. In our case study, stakeholders had significant commitment and willingness to collaborate, because we adjusted the health check-up activities to their needs and priorities. During Phase 2, we conducted a needs assessment using a survey interview. More than one-third of participants reported needing eye check-ups, which more than half reported utilizing Ayurvedic medicine when sick. Based on the outcomes of this needs assessment, we incorporated an eye exam into the check-up, and assigned Indian doctors with knowledge of Ayurveda to the PHC health consultation services. As a result, local stakeholders and participants expressed strong interest and willingness to cooperatively design and implement our research activities. Moreover, based on the results of the needs assessment and multiple focus group discussions, we learned about the gap between top-down, scientific, expert knowledge, and bottom-up, practical knowledge from community members. We found that research activities based on bottom-up, practical knowledge and the needs of local participants increased the satisfaction of both local participants stakeholders, increasing their willingness to collaborate in the project. In addition, the use of innovative and accurate tests and devices for check-ups correlated with the needs of local stakeholders, thus facilitating effective co-production among them. In Phase 3 , we used innovative procedures such as eye exams and hemoglobin tests, which were imported from Germany and Japan, respectively. In the research evaluation of Phase 3, local stakeholders mentioned their appreciation for these new tests, and that they trusted the accuracy of the results. They also noted their desire to continue implementing these activities on a regular basis. Meanwhile, the participants were very curious about the new tests, increasing their overall satisfaction with the health check-up services.

Fourth, we found that the quality of health check-up services must correspond to the needs of local stakeholders, thereby increasing their satisfaction and sense of benefitting from the project. 
We also learned that sufficient training and the use of skilled, well-trained research staff are important for co-production with local stakeholders. As a result of extensive three-day training session, our field staff-including healthcare workers and interviewers-acted in a professional, consistent manner during Phase 3. They also gained further experience in the course of the project, thus further improving their skills. As noted, the use of accurate tests and the high quality of services with well-trained research staff helped increase local stakeholder trust, as did attention to local needs (Figure 9).

Fifth, we realized the importance of trust and the necessity of effective coordinators. From the beginning of co-design, there was good rapport between our local stakeholders and the BGC, because the BGC is widely known as a non-profit organization that contributes to local community development, social services, and women's empowerment. With such a positive reputation and pre-established networks, the BGC served as an effective local coordinator and intermediary between local stakeholders and KU. As such, the fifth lesson and key factor pertains to effective coordinators who are trusted by the local communities and are thus able to utilize and expand their networks to advance co-design and co-production.

Sixth, we recognized the role played by the personalities and characteristics of stakeholder leaders. Characterized by social interaction, positive engagement, and an action-oriented nature, our stakeholders were mostly willing, supportive, and enthusiastic to try new things in healthcare services. Such openness, agreeableness, and positive stakeholder characteristics proved favorable for effective co-design and co-production.

Seventh, we learned that the capacity building of field research staff and the empowerment of local participants is a key factor in successful co-design and co-production. In this study, all of the field staff were hired from the BGC senior student pool and faculty who were interested in local, community-based health research. Throughout the training and research activities, these staff members acquired knowledge, skills, and self-confidence pertaining to health check-ups, disease prevention, and health information. In addition to the staff, local participants gained awareness of their personal health conditions after receiving check-ups and test results. As a result of capacity-building and the empowerment of staff and participants, local stakeholders were more willing to collaborate in the research activities.

Finally, we learned that co-design and co-production are time-consuming and need iterations over the course. Therefore, ongoing and consistent effort to engage with stakeholders through meetings, consultations, and actual implementation (the eighth factor) are necessary to maintain collaborative relationships. We found that more direct, in-person courtesy visits, meetings, and workshops-rather than phone calls, Skype, and email exchanges—-were more effective for communicating with local stakeholders. 


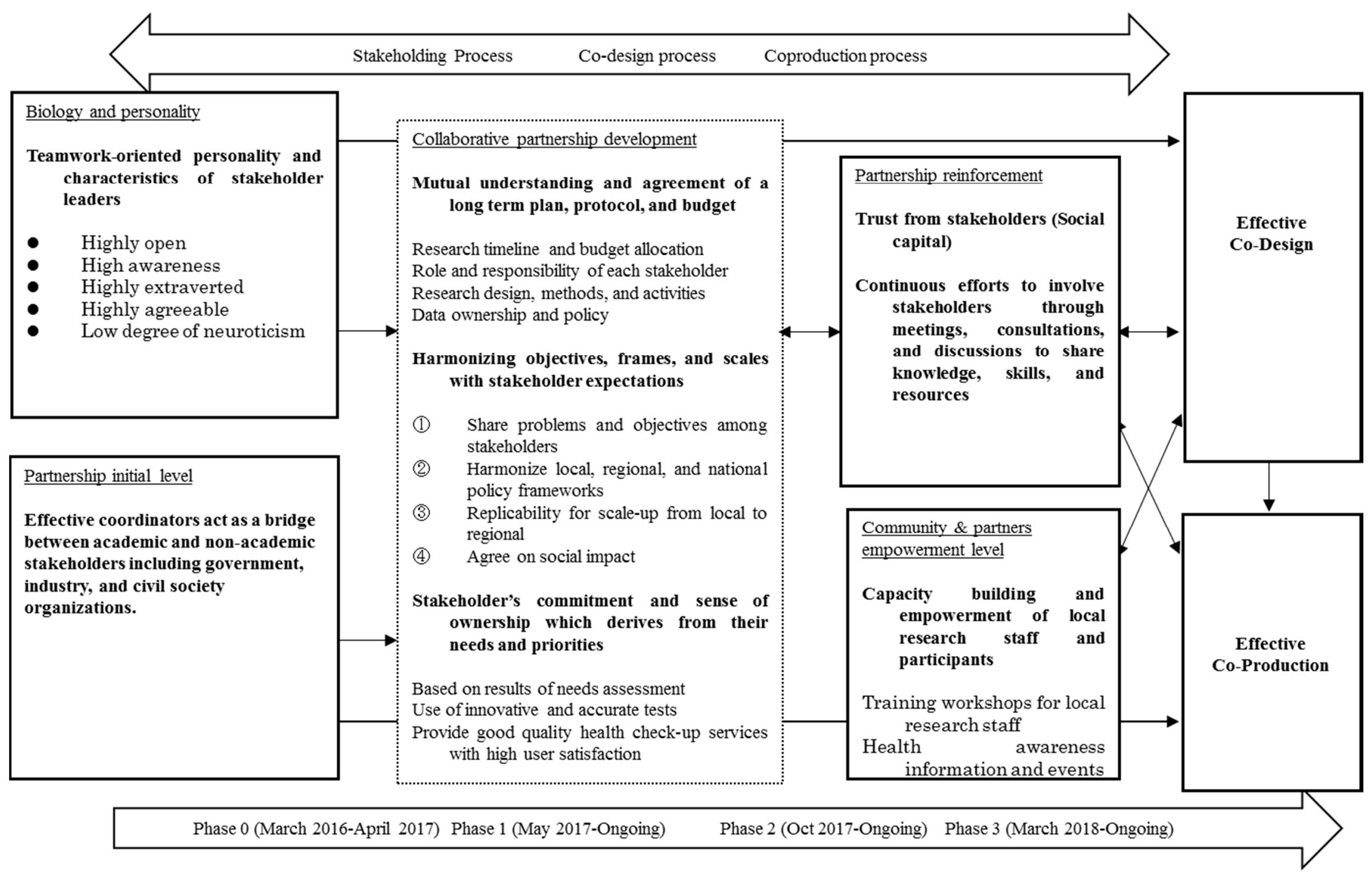

Figure 9. Conceptual framework: key factors that effectively influenced co-design and co-production in the PHC research project in India. 


\section{Discussion}

In this health-focused, transdisciplinary study, we learned that the following eight factors facilitate effective co-design and co-production processes: (1) mutual stakeholder agreement on a long-term research plan, protocol, and budget; (2) correlating research objectives, frames, and the scale of stakeholder expectations; (3) stakeholder commitment and cultivating a sense of ownership by adapting to their needs and priorities; (4) stakeholder trust; (5) effective coordinators; (6) stakeholder leader's personality type and characteristics; (7) capacity-building and the empowerment of local research staff and participants; and (8) ongoing efforts to involve stakeholders throughout co-design and co-production. A number of studies have documented the lessons learned, principles, and frameworks of the early co-design phases in transdisciplinary research $[1,2,4,5,7,9,12-19,28]$. These include the "framing of problems and objectives", "social capital", "joint understanding and mutual trust", "scaling", "accountability", "ownership and roles", "priorities and needs", and "capacity-building". Many of these key factors are similar to those identified in our PHC research in India. As such, it is necessary to discuss these key factors in relation to the extant literature.

With regard to the first key factor-“"mutual stakeholder agreement on a long-term research plan, protocol, and budget" -Naqshbandi et al. have suggested the importance of a flexible research agenda amendable to community timelines, as well as need to ensure that stakeholder roles and research methodology are mutually agreed upon [28]. In addition, Zscheischler et al. reported that transdisciplinary research is a science-practice collaboration with both scientific and societal actors jointly controlling the research plan, design, and protocol-that is to say, the research is not only controlled by researchers [12]. The findings of these previous studies support the notion that our first key factor is critical for effective co-design and co-production. Furthermore, Moser has emphasized that a sense of co-ownership on the part of local stakeholders is key in the initial stage of co-design [1]. This indicates that mutual understanding of and agreement on stakeholder roles and responsibilities under the research plan, protocol, and budget will positively influence stakeholders' sense of ownership.

The second key factor that we identified-“correlating research objectives, frames, and the scale of stakeholder expectations" - was indicated in the work of Emmons et al., who demonstrated that developing shared expectations, operating principles, and language in the context of academiccommunity partnerships is vital [15]. Page et al. have also stressed the importance of careful research design that includes a research purpose, vision, and incentives that motivate stakeholders with different perspectives and backgrounds [7]. These studies support our claim that incorporating research objectives, frames, and scales that correlate with stakeholder perspectives and expectations is necessary for co-design and co-production.

The third key factor- "stakeholders' commitment and a sense of ownership derived from their needs and priorities" - has been illustrated by Van der Hel et al. These researchers reported that transdisciplinary research - which is responsive to the needs of societal actors-is essential for developing social credibility and accountability, thereby increasing the likelihood of collaboration between science and society [9]. Emmons has also suggested that transdisciplinary research in community settings requires knowledge of local cultural needs and preferences [15]. These findings indicate that transdisciplinary research, which is based on stakeholder needs and priorities, can encourage stakeholder commitment, a sense of ownership, and accountability for co-design and co-production. Moreover, Van der Hel et al. indicated that this third factor develops and increases "stakeholder trust and accountability" (i.e., social capital), which is the fourth key factor.

Regarding stakeholder trust and social capital—the fourth key factor-Naqshbandi et al. proposed that "promoting dialogue and developing trust between all partners" is a key principle of CBPR [28] (p. 407). Zscheischler et al. also underscored the importance of mutual trust and network reliance among project partners [12]. As such, these studies support our finding that stakeholder trust and accountability are helpful for effective co-design and co-production.

The fifth key factor is the necessity of an effective coordinator to act as a bridge between academic and non-academic stakeholders. The BGC has extensive networks and a long history 
of collaborating with community organizations, industries, and different levels of government in Rajasthan. Coordinators who understand local situations and are trusted by local communities are critical for the initiative overall, not just effective co-design and co-production [15]. We found that effective coordinators can help facilitate and harmonize different understandings, expectations, and needs among stakeholders. This indicates that the fifth factor is deeply related to the first, second, and third key factors. In this regard, Zscheischler et al. (2017) have highlighted the importance of coordinating researchers who can facilitate joint decision-making and mutual learning, not only between science and practice, but also between the natural and social sciences over the course of transdisciplinary research projects [12].

The sixth key factor we identified pertained to "stakeholder leader's personality type and characteristics." Moser reported that the openness, agreeableness, and positive characteristics of stakeholders were critical elements for effective communication and knowledge sharing. Moreover, Yahara illustrated that good leadership comprises five personality characteristics, in which an individual is: (1) highly open; (2) highly aware; (3) highly extroverted; (4) highly agreeable; and (5) not overly neurotic and acts with clear vision, action, and compassion [29]. As such, the extant literature indicates that co-design and co-production run more smoothly if stakeholder leaders have the following five characteristics: (1) a willingness to try new and unconventional things; (2) willingness to plan, organize, and take responsibility; (3) be socially engaged, enthusiastic, and action-oriented; (4) be friendly, co-operative, compassionate, and less suspicious of others; and (5) feel positive emotions, be slow to anger, and exhibit emotional stability.

The seventh key factor is "capacity-building and the empowerment of local research staff and participants". Previous studies have reported that community empowerment and resource development-identified as "co-learning" activities with all of the partners in all of the phases of research—are a key principle in effective transdisciplinary research $[1,5,18]$ as well as CBPR $[15,27,30]$. In most transdisciplinary research, community members involved in research projects are considered local stakeholders. However, markedly few studies have implemented "empowerment" activities within the transdisciplinary process [2]. Real empowerment requires commitment from all social groups, including scientists, policymakers, and civil society [2]. While commitment from local stakeholders was achieved in our case study, not all of the stakeholders were directly involved in capacity-building and empowerment activities-such as joint training sessions and workshops among KU and BGC research staff. In our research, we found that the more time we spent on capacity-building, training, and empowerment activities prior to implementation, the better the quality of the PHC research activities and the more satisfied the participants.

Finally, the eighth factor that we identified as integral is the need for "ongoing efforts to involve stakeholders throughout the co-design and co-production processes". In this regard, Zscheischler et al. suggested that regular meetings between science and practice resulted in mutual learning, collaboration, and increased motivation among stakeholders [12]. In addition, Emmons et al. and Naqshbandi et al. noted that sharing knowledge and information through multidirectional communication with community partners in all of the phases of research is helpful for sustainable collaboration and partnerships [15,28]. Naqshbandi et al. also suggested that face-to-face communication by traveling to meet with community leaders is important to build and maintain strong relationships and partnerships with stakeholders. In our study, we made multiple courtesy visits and held meetings and discussions before, during, and after implementing the PHC research. We usually provided lunch, snacks, and/or coffee during the meetings. These findings suggest that continuous efforts toward direct face-to-face meetings facilitated the building of trust and mutual understanding among stakeholders, thereby supporting the other factors.

Although the eight factors discussed in the previous section have facilitated effective co-design and co-production in our research, we experienced several challenges. For example, we experienced communication difficulties due to different expectations, priorities, and values among non-academic stakeholders, as well as the complexity of the local society. Oftentimes, it was hard to bridge these 
gaps between scientific theory and real-world practice. Structures of co-production are inherently unstable and may have unclear and/or shifting goals, and this process also produces conflicts and can be unpredictable among stakeholders [3]. In retrospect, we should have engaged with all levels of government, industry, and communities earlier in our co-design process; this would have reduced the need to go back and forth between co-design and co-production. In addition, we should have involved more people from all levels of the organizational hierarchy among each stakeholder to harmonize the gaps within each stakeholder.

Certain key factors-such as effective local coordinators, the personality types of stakeholder leaders, and ongoing involvement with stakeholders-helped mitigate challenging situations.

\section{Conclusions}

Based on the lessons learned from our case study - the development and implementation of PHC services in India-we identified eight factors that were useful for effective co-design and co-production. Although these factors are similar to those described in previous studies, the frameworks developed according to these eight factors (Figure 9) provide insight into successful co-design and co-production in the context of health-related, transdisciplinary research in Asia. Additionally, this paper suggests the potential of the community-based, mobile health check-up project in Jaipur, which will contribute to SDGs such as "good health and well-being" (SDG3) and "partnerships for goals" (SDG17) by encouraging more community people, industry, and government employees to have health check-ups on a regular basis.

Author Contributions: Study conceptualization, F.Y. and M.B.; Methodology, F.Y.; System software, A.A., R.I. and Y.N.; Training, R.I., M.N., and Y.N.; Validation, F.Y. and M.B.; Formal analysis, M.N., Y.F.; Investigation, F.Y., M.B., M.N., Y.N.; Writing—original draft preparation, F.Y.; Writing-review and editing, M.B., A.A., R.I., M.N., and K.K.; Supervision, A.A., R.I., and N.N.; Project administration, M.B.; Funding acquisition, N.N., M.N., A.A., and F.Y.

Funding: This research was funded by the Future Earth Research Fund, grant number 18-161009264; and the Kyushu University QR Program, grant number 30105.

Acknowledgments: I would like to thank following stakeholders and the individuals who provided supports in the project; Biyani group of colleges, Grameen Communications, Kalwar village office, Kalwar health center, Jaipur District and Rajasthan state department of medical, health, and family welfare, Brahma Kumaris Spiritual Centers, and Saras Dairy, India. Special thanks to Yasunori Hanamatsu at Kyushu International University, Takahiro Fujiwara at Kyushu University, Department of Agriculture, Tetsukazu Yahara, Nariaki Onda, Tatsuro Sato, Tomomi Yamashita, Wataru Tanaka at Kyushu University, Institute of Decision Science and Sustainable Society, who provided valuable advice on the project.

Conflicts of Interest: The authors declare no conflict of interest.

\section{References}

1. Moser, S.C. Can science on transformation transform science? Lessons from co-design. Curr. Opin. Environ. Sustain. 2016, 20, 106-115. [CrossRef]

2. Brandt, R.; Ernst, A.; Gralla, F.; Luederitz, C.; Lang, D.J.; Newig, J.; Reinert, F.; Abson, D.J.; von Wehrden, H. A review of transdisciplinary research in sustainability science. Ecol. Econ. 2013, 92, 1-15. [CrossRef]

3. Greenhalgh, T.; Jackson, C.; Shaw, S.; Janamian, T. Achieving Research Impact through Co-creation in Community-Based Health Services: Literature Review and Case Study. Milbank Q. 2016, 94, $392-429$. [CrossRef] [PubMed]

4. Leemans, R. The lessons learned from shifting from global-change research programmes to transdisciplinary sustainability science. Curr. Opin. Environ. Sustain. 2016, 19, 103-110. [CrossRef]

5. Scholz, R.W.; Steiner, G. The real type and ideal type of transdisciplinary processes: Part I-Theoretical foundations. Sustain. Sci. 2015, 10, 527-544. [CrossRef]

6. Future Earth. Future Earth Engagement Principles and Practice. 2016. Available online: http:/ futureearth. org/sites/default/files/futureearth_engagementprinciplespractice_2016.pdf (accessed on 3 May 2018).

7. Page, G.G.; Wise, R.M.; Lindenfeld, L.; Moug, P.; Hodgson, A.; Wyborn, C.; Fazey, L. Co-designing transformation research: Lessons learned from research on deliberate practices for transformation. Curr. Opin. Environ. Sustain. 2016, 20, 86-92. [CrossRef] 
8. Scholz, R.W.; Lang, D.J.; Wiek, A.; Walter, A.I.; Stauffacher, M. Transdisciplinary case studies as a means of sustainability leaning. Historical framework and theory. Int. J. Sustain. High. Educ. 2006, 7, 226-251. [CrossRef]

9. Van Del Hel, S. New science for global sustainability? The institutionalization of knowledge co-production in Future Earth. Environ. Sci. Policy 2016, 61, 165-175. [CrossRef]

10. Lövbrand, E.; Beck, S.; Chilvers, J.; Forsyth, E.; Hedren, J.; Hulme, M.; Lidskog, R.; Vasileidou, E. Who speaks for the future of earth? How critical social science can extend the conversation on the Anthropocene. Glob. Environ. Chang. 2015, 32, 211-218. [CrossRef]

11. Future Earth. Future Earth 2025 Vision. 2014. Available online: http://www.futureearth.org/sites/default/ files / future-earth_10-year-vision_web.pdf (accessed on 3 May 2018).

12. Zscheischler, J.; Rogga, S.; Busse, M. The adoption and implementation of transdisciplinary research in the field of land-use science: A comparative case study. Sustainability 2017, 9, 1926. [CrossRef]

13. Adler, C.; Hadorn, G.H.; Breu, T.; Wiesmann, U.; Pohl, C. Conceptualizing the transfer of knowledge across case in transdisciplinary research. Sustain. Sci. 2018, 13, 179-190. [CrossRef] [PubMed]

14. Mckee, A.; Guimaraes, M.H.; Pinto-Correia, T. Social capital accumulation and the role of the searcher: An example of a transdisciplinary visioning process for the future of agriculture in Europe. Environ. Sci. Policy 2015, 50, 88-99. [CrossRef]

15. Emmons, K.M.; Viswanath, K.; Colitz, G.A. The role of transdisciplinary collaboration in translating and disseminating health research. Am. J. Prev. Med. 2008, 35, S204-S210. [CrossRef] [PubMed]

16. Ruddy, G.; Rhee, K. Transdisciplinary teams in primary care for the underserved: A literature review. J. Health Care Poor Underserved 2005, 16, 248-256. [CrossRef] [PubMed]

17. Fraser, E.D.G.; Dougill, A.J.; Mabee, W.E.; Reed, M.; McAlpine, P. Bottom up and top down: Analysis of participatory processes for sustainability indicator identification as a pathway to community empowerment and sustainable environmental management. J. Environ. Manag. 2006, 78, 114-127. [CrossRef] [PubMed]

18. Lang, D.J.; Wiek, A.; Bergmann, M.; Stauffacher, M.; Martens, P.; Moll, P.; Swilling, M.; Thomas, C.J. Transdisciplinary research in sustainability science: Practice, principles, and challenges. Sustain. Sci. 2012, 7 , 25-43. [CrossRef]

19. Rhodes, S.D.; Kelley, C.; Siman, F.; Cashman, R.; Alonzo, J.; McGuire, J.; Wellendorf, T.; Hinshaw, K.; Allen, A.B.; Downs, M.; et al. Using community-based participatory research (CBPR) to develop a communitylevel HIV prevention Intervention for Latinas: A local response to a global challenge. Womens Health Issues 2012, 22, E293-E301. [CrossRef] [PubMed]

20. Rosenfield, P.L. The potential of transdisciplinary research for sustaining and extending linkages between and the health and social sciences. Soc. Sci. Med. 1992, 35, 1343-1357. [CrossRef]

21. Grameen Foundation Website. Grameen Family of Organizations. Available online: https://grameenfound ation.org/about/related-organizations/grameen-family-organizations (accessed on 29 May 2018).

22. Nohara, Y.; Kai, E.; Ghosh, P.P.; Islam, R.; Ahmed, A.; Kuroda, M.; Inoue, S.; Hiramatsu, T.; Kimura, M.; Shimizu, S.; et al. Health checkup and telemedical intervention program for preventive medicine in Developing Countries: Verification Study. J. Med. Internet Res. 2015, 17, e2. [CrossRef] [PubMed]

23. Ahmed, A.; Rebeiro-Hargrave, A.; Nohara, Y.; Kai, E.; Hossein Ripon, Z.; Nakashima, N. Targeting Morbidity in Unreached Communities Using Portable Health Clinic System. IEICE Trans. Commun. 2014, E97-B, 540-545. [CrossRef]

24. Nakashima, N.; Nohara, Y.; Ahmed, A.; Kuroda, M.; Inoue, S.; Ghosh, P.P.; Islam, R.; Hiramatsu, T.; Kobayashi, K.; Inoguchi, T.; et al. An affordable, usable and sustainable preventive healthcare system for unreached people in Bangladesh. Stud. Health Technol. Inform. 2013, 192, 1051. [CrossRef] [PubMed]

25. Nakashim, N.; Hiramatsu, T.; Ghosh, P.P.; Islam, R.; Kobayashi, K.; Inoguchi, T. Evaluation of "Portable Health Clinic" with BAN standard for $10 \mathrm{~K}$ subjects in Bangladesh. Conf. Proc. IEEE Eng. Med. Biol. Soc. 2013, 2013, 1672-1675.

26. Grameen Communications. Global Communication Center Portable Health Clinic. 2018. Available online: http:/ / ghealth.gramweb.net/ (accessed on 6 May 2018).

27. Brahma Kumaris Website. Brahma Kumaris. 2018. Available online: http://www.brahmakumaris.org/8-iw eb /262-en-gb-brahma-kumaris-world-spiritual-university (accessed on 6 May 2018). 
28. Naqshbandi, M.; Harris, S.B.; Macaulay, A.C.; Comeau, J.; Piche, J.; Montour-Lazare, D. Lessons learned in using community-based participatory research to build a national diabetes collaborative in Canada. Prog. Community Health Partnersh. 2011, 5, 405-415. [PubMed]

29. Yahara, T. An Introduction to Decision Science: How Can We Transform Our Society to a Sustainable Future; Bun-ichi Publication: Tokyo, Japan, 2017.

30. Israel, B.A.; Schulz, A.J.; Parker, E.A.; Becker, A.B. Review of community-based research: Assessing partnership approach to improve public health. Annu. Rev. Public Health 1998, 19, 173-202. [CrossRef] [PubMed]

(c) (C) 2018 by the authors. Licensee MDPI, Basel, Switzerland. This article is an open access article distributed under the terms and conditions of the Creative Commons Attribution (CC BY) license (http://creativecommons.org/licenses/by/4.0/). 